\title{
Epidemiological Characteristics of Re-emerging Vivax Malaria in the Republic of Korea (1993-2017)
}

\author{
Young Yil Bahk', Hyeong-Woo Lee ${ }^{2}$, Byoung-Kuk Na ${ }^{3}$, Jeonga Kim4, Kyoung Jin ${ }^{5}$, Yeong Seon Hong ${ }^{5}$, \\ Tong-Soo Kim,** \\ ${ }^{1}$ Department of Biotechnology, College of Biomedical and Health Science, Konkuk University, Chungju 27478, Korea; ${ }^{2}$ nnsitute of Research and \\ Development, Scorpiogen Co., Hankyong National University, Anseong 17579, Korea; ${ }^{3}$ Department of Parasitology and Tropical Medicine and \\ Institute of Health Sciences, Gyeongsang National University College of Medicine, Jinju 52727, Korea; ${ }^{4}$ Department of Medicine, Division of \\ Endocrinology, Diabetes, and Metabolism, UAB Comprehensive Diabetes Center, University of Alabama at Birmingham, Birmingham, AL 35294, \\ USA; ${ }^{5}$ Korea Health Evaluation Institute, Sahmyook University, Seoul 01795, Korea; ${ }^{6}$ Department of Parasitology and Tropical Medicine, Inha \\ University School of Medicine, Incheon 22212, Korea
}

\begin{abstract}
Historically, Plasmodium vivax malaria has been one of the most highly endemic parasitic diseases in the Korean Peninsula. Until the 1970s, vivax malaria was rarely directly lethal and was controlled through the Korean Government Program administered by the National Malaria Eradication Service in association with the World Health Organization's Global Malaria Eradication Program. Vivax malaria has re-emerged in 1993 near the Demilitarized Zone between South and North Korea and has since become an endemic infectious disease that now poses a serious public health threat through local transmission in the Republic of Korea. This review presents major lessons learned from past and current malaria research, including epidemiological and biological characteristics of the re-emergent disease, and considers some interesting patterns of diversity. Among other features, this review highlights temporal changes in the genetic makeup of the parasitic population, patient demographic features, and spatial distribution of cases, which all provide insight into the factors contributing to local transmission. The data indicate that vivax malaria in Korea is not expanding exponentially. However, continued surveillance is needed to prevent future resurgence.
\end{abstract}

Key words: Plasmodium vivax, re-emerging malaria, control, mosquito, vector

\section{INTRODUCTION}

Malaria is one of the oldest known and most prevalent parasitic diseases in the world, with an estimated 3.2 billion people being at risk of infection [1]. The incidence of malaria has decreased in recent years. In 2016, there were an estimated 216 million cases of malaria, about 5 million more cases than in 2015, and the number of deaths reached 445,000, a similar number to the previous year [2]. Among the protozoa that cause malaria, Plasmodium falciparum is the greatest menace because of its high mortality rate. Malaria caused by $P$. vivax is less lethal but is a huge burden on public health, greatly affecting the quality of life for many people in tropical, subtropical, and temperate countries $[3,4]$. Previous eradication programs

- Received 12 April 2018, revised 29 October 2018, accepted 2 November 2018.

*Corresponding author (tongsookim@inha.ac.kr)

(c) 2018, Korean Society for Parasitology and Tropical Medicine

This is an Open Access article distributed under the terms of the Creative Commons Attribution Non-Commercial License (http://creativecommons.org/licenses/by-nc/4.0) which permits unrestricted non-commercial use, distribution, and reproduction in any medium, provided the original work is properly cited. have shown that $P$. vivax can persist long after $P$. falciparum is eliminated [5]. Unlike P. falciparum, P. vivax infections include dormant hypnozoites that can survive in the liver without the onset of disease and cause clinical relapses. These relapses manifest as blood infections that are clinically indistinguishable from primary infections. They constitute a substantial, but geographically variable, proportion of the total infections and disease risk within populations [6,7]. Moreover, parasitemia in a $P$. vivax infection is usually of a lower density than that in a $P$. falciparum infection, making diagnostic tools less effective.

Vivax malaria has been an endemic infectious disease in the Korean Peninsula for a long time (Fig. 1). Indigenous falciparum malaria has not been reported. vivax malaria was eliminated in Korea in the late 1970s but re-emerged in the early 1990 s and has continued to prevail despite the ongoing national eradication program $[8,9]$.

The aim of this brief review is to provide key information on the epidemiological and biological characteristics of reemerging vivax malaria in Korea. The review focuses on the 
characterization of the transmission of vivax malaria in Korea after its re-emergence in 1993.

\section{MALARIA CONTROL UP TO 1993}

In Korea, malaria is classified as a group III infectious disease and malaria control efforts started in the middle of the 20th century. From the early 1970s to 1993, Korea was considered to be a malaria-free region. Historically, however, P. vivax malaria was highly endemic in the Korean Peninsula for centuries and caused unstable malaria transmission $[8,9]$.

Malaria is popularly known as Hah-roo-geo-ri (meaning "every-other-day fever") or Hahk-geel (Korean disease name) [10]. Medical characteristics of vivax malaria in Korea include few previous clinical malaria episodes prior to the current infection, no association with concurrent bacteremia, and extremely rare occurrences of severe anemia, hypoglycemia, and acute kidney injury complications. Mortality is rare. Relatively common and notable characteristics include cerebral and pulmonary manifestations, spontaneous bleeding, shock, and metabolic acidosis [11]. Many medical books published in the Joseon Dynasty described the main symptoms and treatments for the disease. The first systematic and scientific descriptions of malaria in the Korean Peninsula were published in 1913 [12]. The article described $P$. vivax cases confirmed by micros- copy in the general population and stationed Japanese army soldiers. The same article also provided information on the prevalence of vivax malaria in the Korean Peninsula during the Japanese colonial era. During the Korean War (1950-1953), vivax malaria became a serious problem. Treatment of American troops infected with vivax malaria and the recurrence of the infection after repatriation were important medical issues $[13,14]$. Malaria control was consolidated in 1959 with the inauguration of the National Malaria Eradication Service (NMES) under the control of Korean Government with the assistance of the Global Malaria Eradication Program of the World Health Organization (WHO), which included regions of the globe other than Africa. As a part of these efforts, a nationwide survey was performed to gauge the prevalence of the protozoan parasite [15]. The use of effective insecticides and anti-malarial drugs, as well as an improved standard of living and easy access to medical clinics facilitated the effective treatment of malaria. As a consequence, the national prevalence of malaria was markedly diminished by the late 1970s (Fig. 2) [16-19]. Extensive use of agricultural pesticides also contributed greatly to the decrease in malaria incidence through the eradication of the mosquito vector of the disease. In 1979, the WHO officially certified that Korea was a malaria-free country. The country was the first nation to receive this WHO certification [20]. With the exception of a memoir citing 2 cases of

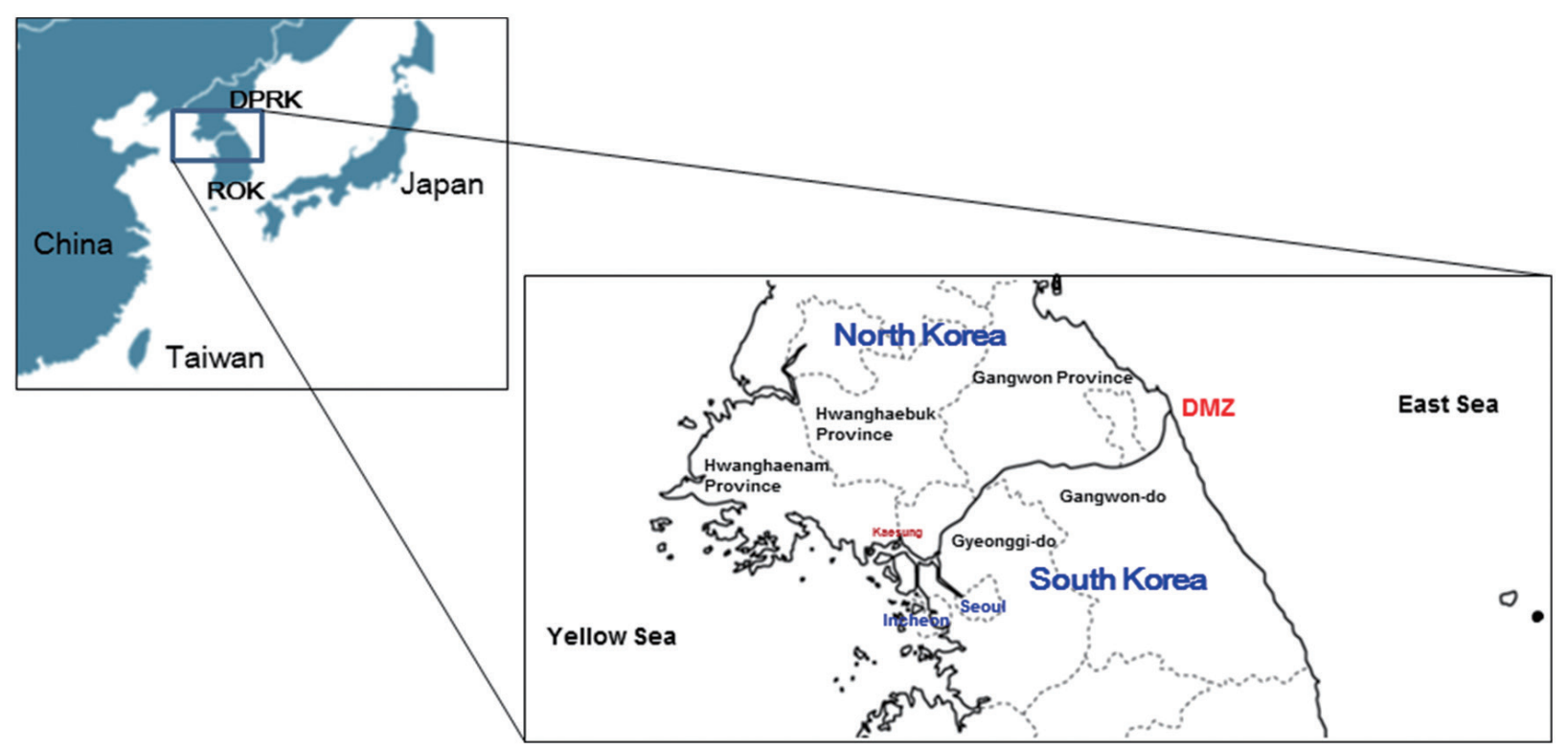

Fig. 1. Map of the Korean Peninsula. The DMZ divides Korea and North Korea. Malaria in Korea occurs mainly in the northern area close to the DMZ of Incheon-si, Gyeonggi-do, and Gangwon-do, and malaria in North Korea occurs in Gaeseong City, North and South Hwanghae provinces, and Gangwon province. 
malaria in 1985 [18], no official malaria cases were reported the western edge of the Demilitarized Zone (DMZ), a fortified in Korea until 1993. At that time, one case was reported along zone $4 \mathrm{~km}$ wide and $248 \mathrm{~km}$ long that incorporates territory

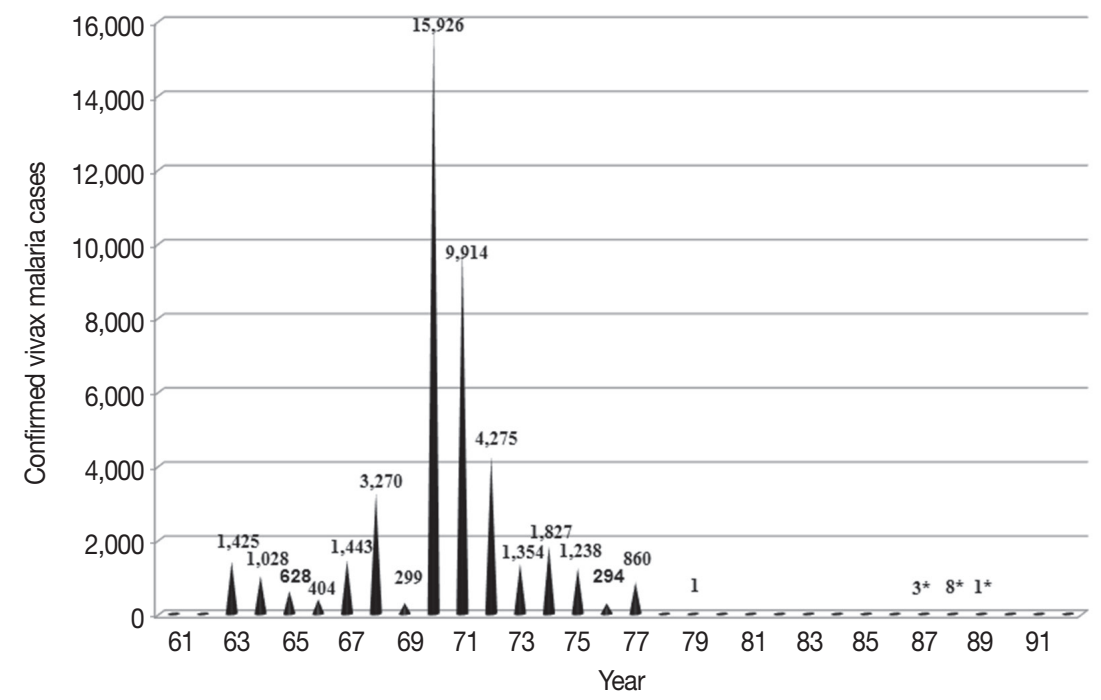

Fig. 2. Indigenous malaria cases in Korea before re-emergence. Indigenous vivax malaria was highly prevalent in Korea, but it was successfully eradicated from the region in the early 1970s [13-16]. The asterisk indicates the number of imported cases of malaria.

Table 1. Number and percentage of vivax malaria cases confirmed in Korea from 1993 through 2017, and the distribution of the confirmed cases according to the month

\begin{tabular}{|c|c|c|c|c|c|c|c|c|c|c|c|c|c|}
\hline Year & No. & Jan. & Feb. & Mar & Apr. & May & Jun. & Jul. & Aug. & Sep. & Oct. & Nov. & Dec. \\
\hline 1993 & 3 & & 1 & 1 & & & 1 & & & & & & \\
\hline 1994 & 25 & & & 1 & & 1 & 2 & 1 & & & 20 & & \\
\hline 1995 & 107 & & & 1 & & & 13 & 22 & 44 & 22 & 5 & & \\
\hline 1996 & 356 & & & & 2 & 10 & 20 & 57 & 138 & 102 & 24 & 3 & \\
\hline 1997 & 1,724 & 3 & 1 & 4 & 12 & 35 & 163 & 337 & 465 & 519 & 144 & 36 & 5 \\
\hline 1998 & 3,932 & 6 & 8 & 9 & 40 & 164 & 509 & 1,182 & 1,199 & 553 & 219 & 34 & 9 \\
\hline 1999 & 3,621 & 13 & 12 & 22 & 80 & 257 & 516 & 798 & 978 & 602 & 273 & 59 & 11 \\
\hline 2000 & 4,142 & 16 & 14 & 30 & 62 & 285 & 516 & 842 & 1,076 & 862 & 272 & 115 & 52 \\
\hline 2001 & 2,556 & 14 & 19 & 34 & 45 & 176 & 369 & 681 & 624 & 378 & 173 & 30 & 12 \\
\hline 2002 & 1,799 & 11 & 4 & 5 & 34 & 102 & 192 & 407 & 561 & 297 & 145 & 36 & 5 \\
\hline 2003 & 1,171 & 10 & 8 & 9 & 25 & 73 & 176 & 275 & 295 & 186 & 85 & 22 & 7 \\
\hline 2004 & 864 & 6 & 7 & 4 & 19 & 42 & 141 & 211 & 214 & 126 & 76 & 13 & 5 \\
\hline 2005 & 1,369 & 6 & 5 & 12 & 21 & 49 & 133 & 295 & 398 & 256 & 154 & 34 & 6 \\
\hline 2006 & 2,051 & 9 & 3 & 19 & 29 & 109 & 301 & 432 & 516 & 384 & 193 & 40 & 16 \\
\hline 2007 & 2,227 & 15 & 8 & 10 & 20 & 110 & 239 & 605 & 639 & 329 & 173 & 65 & 14 \\
\hline 2008 & 1,052 & 22 & 8 & 8 & 37 & 85 & 148 & 235 & 228 & 172 & 85 & 14 & 10 \\
\hline 2009 & 1,345 & 3 & 3 & 9 & 48 & 121 & 213 & 313 & 269 & 211 & 99 & 45 & 11 \\
\hline 2010 & 1,772 & 1 & 8 & 8 & 36 & 171 & 358 & 454 & 401 & 211 & 93 & 21 & 10 \\
\hline 2011 & 838 & 8 & 10 & 12 & 25 & 82 & 171 & 209 & 166 & 95 & 44 & 12 & 4 \\
\hline 2012 & 555 & 3 & 3 & 6 & 10 & 45 & 89 & 147 & 116 & 81 & 36 & 10 & 9 \\
\hline 2013 & 445 & 4 & 6 & 8 & 15 & 53 & 77 & 95 & 69 & 58 & 49 & 10 & 1 \\
\hline 2014 & 638 & 4 & 10 & 5 & 15 & 28 & 107 & 163 & 142 & 92 & 47 & 10 & 15 \\
\hline 2015 & 699 & 8 & 5 & 8 & 37 & 84 & 129 & 151 & 146 & 79 & 36 & 7 & 9 \\
\hline 2016 & 681 & 9 & 4 & 6 & 30 & 75 & 149 & 166 & 113 & 91 & 35 & 3 & 0 \\
\hline 2017 & 515 & 7 & 7 & 2 & 9 & 37 & 86 & 133 & 112 & 76 & 35 & 4 & 7 \\
\hline $\begin{array}{c}\text { Total } \\
(\%)\end{array}$ & $\begin{array}{c}34,487 \\
(100)\end{array}$ & $\begin{array}{c}178 \\
(0.52)\end{array}$ & $\begin{array}{c}154 \\
(0.45)\end{array}$ & $\begin{array}{c}233 \\
(0.68)\end{array}$ & $\begin{array}{c}651 \\
(1.89)\end{array}$ & $\begin{array}{l}2,194 \\
(6.36)\end{array}$ & $\begin{array}{c}4,818 \\
(13.97)\end{array}$ & $\begin{array}{c}8,211 \\
(23.81)\end{array}$ & $\begin{array}{c}8,909 \\
(25.83)\end{array}$ & $\begin{array}{c}5,782 \\
(16.77)\end{array}$ & $\begin{array}{l}2,515 \\
(7.29)\end{array}$ & $\begin{array}{c}623 \\
(1.81)\end{array}$ & $\begin{array}{l}218 \\
(0.63)\end{array}$ \\
\hline
\end{tabular}


on both South and North Korea. The DMZ was established following the end of the Korean War by pulling back the respective forces $2 \mathrm{~km}$ from each side of the border (Fig. 1) [21,22].

\section{RE-EMERGENCE OF VIVAX MALARIA}

In July 1993, a Korean Army soldier stationed near the western edge of the DMZ in northern Gyeonggi-do (do=province) developed a fever with a periodic pattern of occurrence and was admitted to the Capital Armed Forces General Hospital. He was diagnosed with vivax malaria based on the blood smear method [21], which revealed the typical ring, trophozoite, and gametocyte forms of P. vivax. After this first case of recurrence, there was an exponential increase in vivax malaria cases in Korea with epidemic outbreaks from 1995-2000 (Table 1; Fig. 3). Since then, vivax malaria has been recognized as a notable public health problem [23-25].

Almost all ( $>90 \%$ ) of malaria cases at the beginning of the outbreak occurred among Korean and American soldiers stationed near the DMZ in the northern part of Gyeonggi and Gangwon-do, and among veterans who had been repatriated to their hometowns where they subsequently developed vivax malaria [26]. Prior to 1996, over $80 \%$ of vivax malaria patients were military personnel. However, the prevalence gradually decreased from $65 \%$ in the period of $1997-2000$ (8,786 of the $13,419$ total cases; $[27,28])$ to approximately $40 \%$ in 2007 (908 of the 2,227 total cases) [29]. Correspondingly, the prevalence grew among the civilian population residing in nearby areas. Malaria has once again become endemic and a serious public health burden in Korea. The Korean Army introduced a strategy of mass chemoprophylaxis with chloroquine during the malaria transmission season and primaquine after the transmission season to reduce the number of vivax malaria cases in both the military and civilian populations. Chemoprophylaxis with chloroquine and primaquine began with about 16,000 soldiers in 1997 and was expanded to more than 200,000 military personnel in zones with a high risk for vivax malaria [30].

The increased risk of malaria infection among people near the DMZ suggested that North Korea might be a major reservoir of vivax malaria. The infection would spread across the border to Korea via sporozoite-bearing mosquitoes (Fig. 2) [31]. North Korea had a restricted capacity to eliminate malaria until 1998 and experienced its highest reported incidence of malaria in 2001, peaking at 296,540 microscopically confirmed and indigenous cases (43.4 cases per a population of $1,000)$, according to the 2010 WHO World Malaria Report. Malaria control efforts by North Korea for at-risk populations focused on mass primaquine preventive treatment (MPPT) as

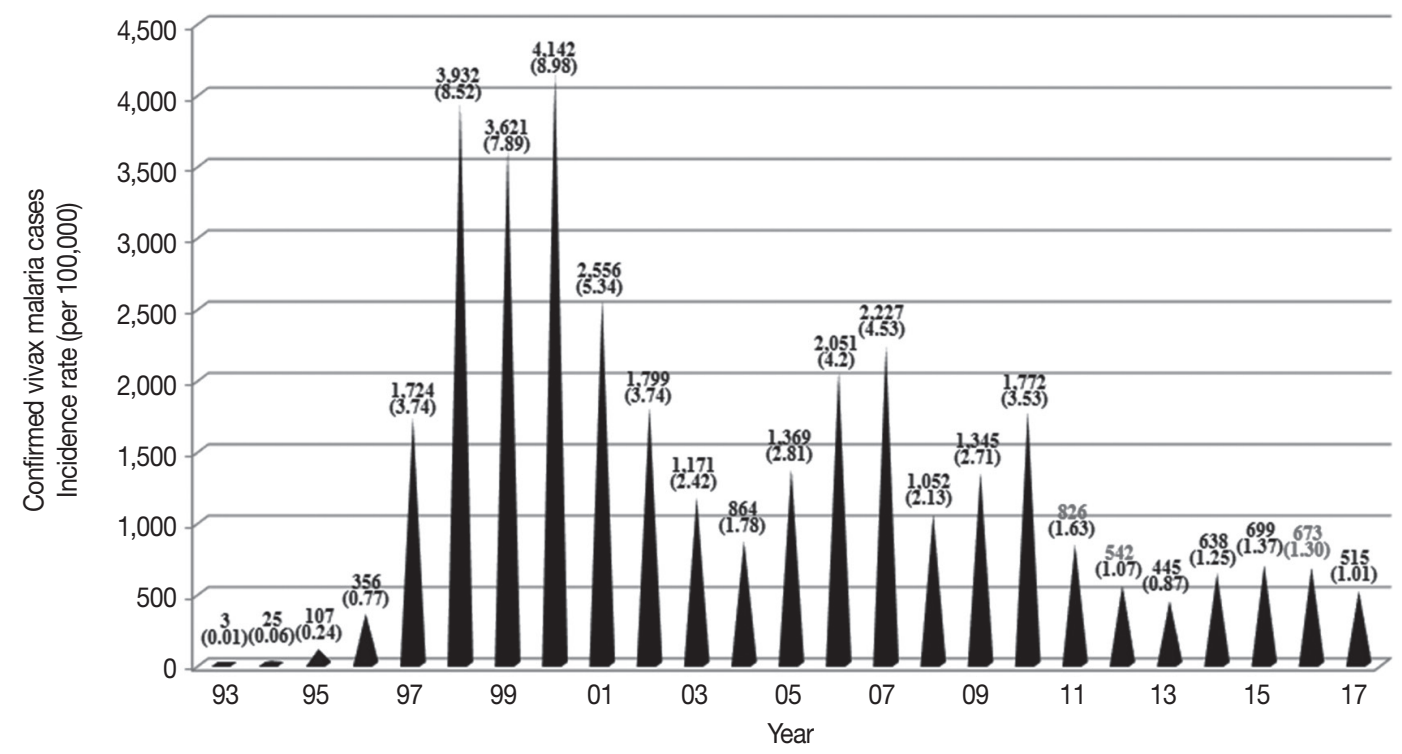

Fig. 3. Indigenous malaria cases in Korea after re-emergence. Since its re-emerging in 1993, the highest patient outbreak occurred in 2000. Since 2000, there has been a mixed pattern of repeated increases and decreases in the incidence of malaria, but the overall trend shows a plateau. 


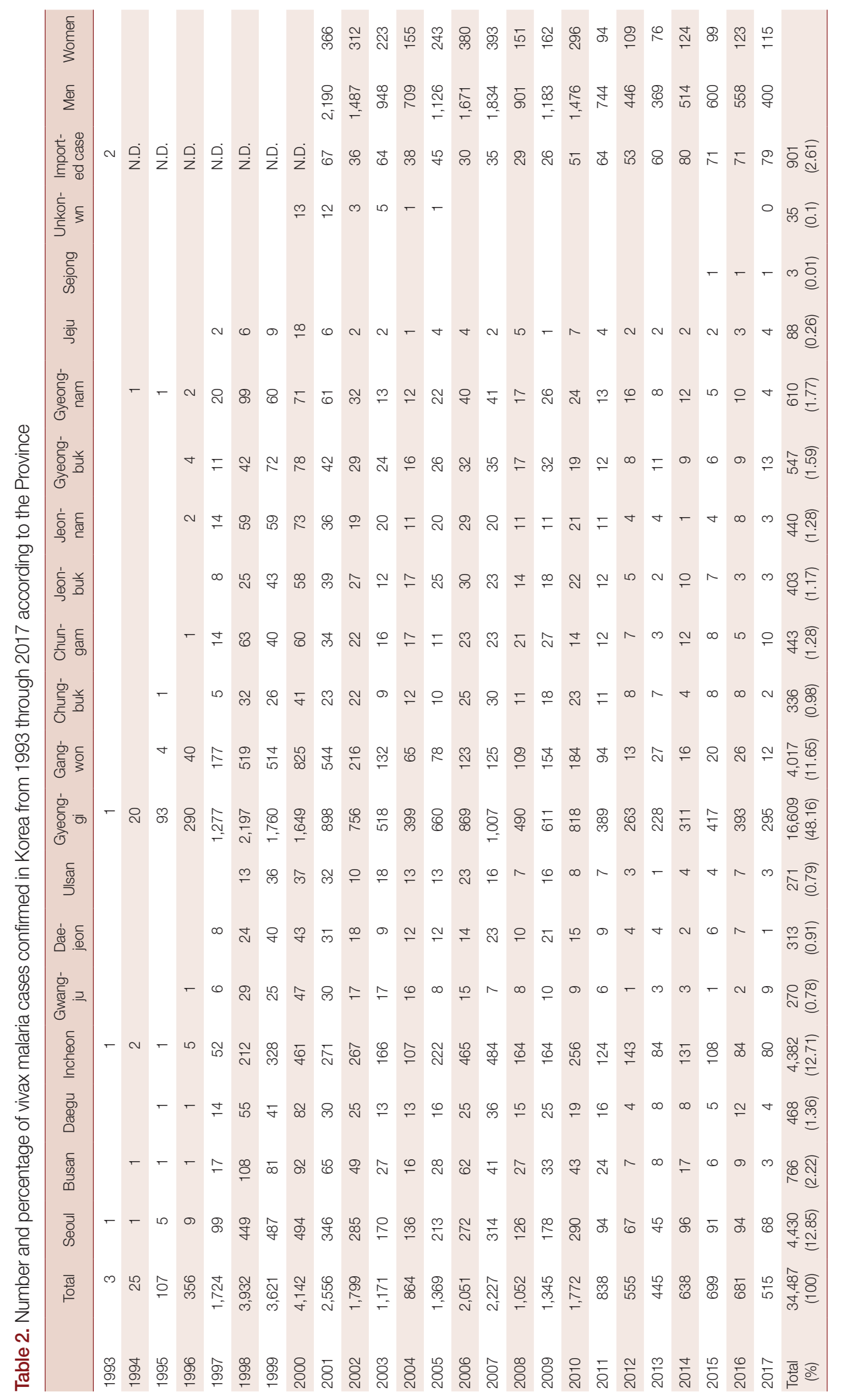




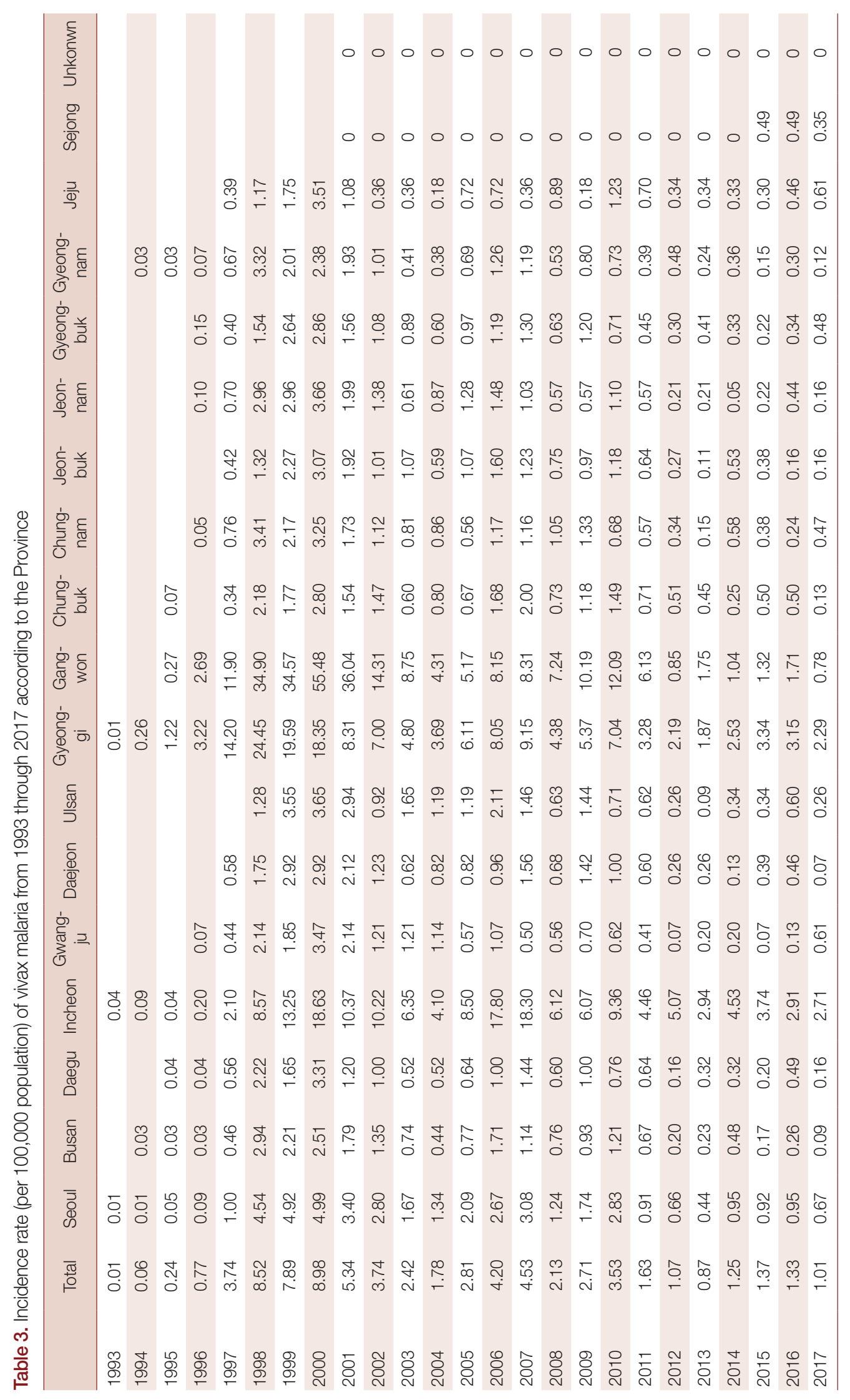


a means of chemoprophylaxis directed towards relapse, and the introduction of a National Malaria Control Program (NMCP) in 1999. Both strategies have been fully operational since 2001 [32,33]. The majority of cases in North Korea occurred in Gangwon and Hwanghae Province, which border the DMZ. Korea had over 4,000 cases reported in the 2010 WHO World Malaria Report (Table 1) [34]. High-risk areas in Korea were adjacent to those in North Korea, including Incheon-si (si=city) and northern Gyeonggi and Gangwondo. Perhaps exacerbating the transmission, travelers from Korea were allowed to visit certain regions of North Korea, including Gaeseong City and Geumgang-san (san=mountain) (Fig. 1). The influx of mosquitoes (Anopheles sinensis) infected with P. vivax from North Korea could be one of the possible routes of re-emergence. An. sinensis, the major anopheline vector for vivax malaria in Korea, travels up to $12 \mathrm{~km}$ in a single night, theoretically permitting traversal of the DMZ [35]. Meanwhile, a haplotype network analysis of the parasite's mitochondrial genome suggested that the genealogical origin of the re-emerged P. vivax in Korea was Southern China [36]. Further complex analyses of the genotypes of the Korean P. vivax population are necessary to conclusively trace the path of reemerged P. vivax in Korea.

\section{PREVALENCE OF THE MALARIA PARASITE}

The published articles included in this review chronicle the prevalence of vivax malaria between 1993 and 2017 in Korea. We incorporated data from several sources, including [1] national-level data including guidelines, reports, documents, and maps from the Korea Centers for Disease Control \& Prevention (KCDC), [2] related data from outside the Ministry of Health and Welfare, [3] scientific articles on malaria in Korea, and [4] WHO reports of technical missions, records, and reports of the WHO Regional Office for Asia meetings. In Korea, reports to the local Public Health Center (PHC) for malaria cases in hospitals or clinics are required by law. PHCs must periodically report cases to the KCDC using the infectious diseases surveillance system [37]. Since the re-emergence of vivax malaria in Korea (1993-2017), 33,972 cases have been diagnosed and reported (Table 1).

The high-risk areas in Korea adjacent to the malaria-risk areas in North Korea, which include Incheon-si, Gyeonggi-do, and Gangwon-do, account for over $70 \%$ of all vivax malaria cases in Korea (Table 2). Each malaria case in Korea is reported in the metropolitan area/province where the diagnosis is made, which may differ from the area where the infection occurred. In addition, it is particularly difficult to ascertain the transmission sites, since approximately $60 \%$ of vivax malaria in Korea is latent, with symptoms occurring 1-24 months after infection [38]. The issue of latency of vivax malaria will be discussed in later sections. Thus, despite the recent progress to reduce malaria cases, these efforts will likely not be sufficient to eliminate malaria from Korea.

The temporal frequency of cases includes 3 major peaks in 2000 (4,142 cases), 2007 (2,227 cases), and 2010 (1,772 cases) (Table 1). The overall decline in cases may reflect the dedicated anti-malarial chemoprophylaxis that has been carried out on military bases since 1997 and the anti-malarial efforts of the government. The current vivax malaria incidence indicates that exponential growth is not occurring. However, the annual volatility of the pattern of sharp decline since 2000 may also correlate to changes in the environment of the parasite and the mosquito in the endemic areas of Korea.

Although the incidence of malaria is directly affected by the malaria parasites and individuals bitten by infected mosquitoes, climate factors such as temperature, precipitation, humidity, rainfall, and flooding also play critical roles [39]. The correlation between climate change and increased malaria risk in Korea is under investigating. However, there are concerns that changing climate determinants will have an impact on the incidence of malaria. For example, climate change might potentially lead to increased locally-acquired autochthonous transmission by enhancing the growth, survival, and distribution of mosquitoes that can mediate malaria and by changing human activities [39-41]. Thus, efforts to suppress vivax malaria cases should be maintained so that no intrinsic potential for transmission remains.

\section{CASE DISTRIBUTION AND SEASONAL TRENDS}

Cases of vivax malaria were limited and sporadic from 1993-1996 but endemic thereafter (Table 1). The incidence of malaria rapidly increased from $0.01 / 100,000$ population in 1993 to $8.52 / 100,000$ population in 1998 , peaking at 8.98/ 100,000 population in 2000 (Table 3). A total of 34,487 indigenous vivax malaria cases were officially reported in Korea from 1993-2017. Although a substantial decrease in the annual number of malaria cases was observed in recent years, vivax malaria still remains endemic in Korea. 


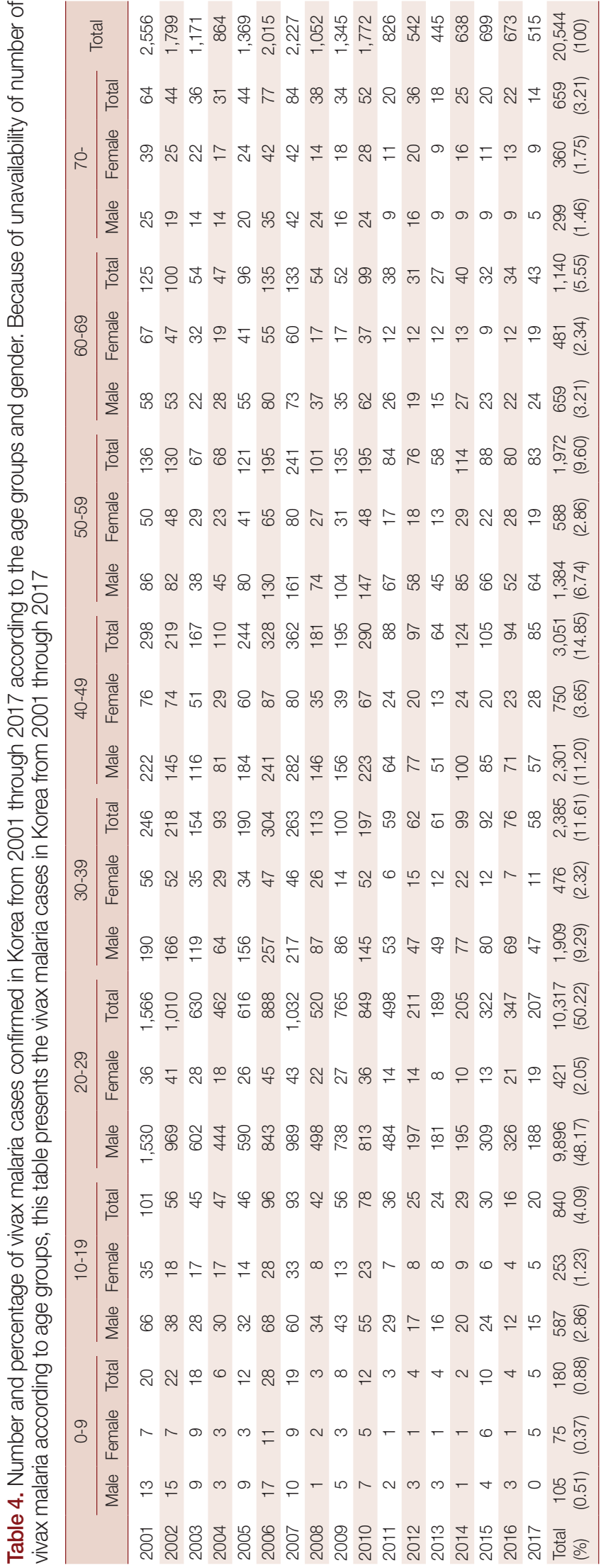

Malaria in Korea is unstable and seasonal, with $94 \%$ of cases (32,429 of 34,487 cases) occurring between 1993-2017 reported between May and October when malaria vectors exist in the field (Table 1). This seasonal distribution pattern of malaria cases has not changed over time and has not been affected by the decline in malaria transmission. Indigenous malaria cases that occurred in the first 4 months of the calendar year, winter and spring, might comprise P. vivax infections contracted in the previous malaria season that had a subsequent prolonged incubation. Such prolonged incubation cases account for approximately $60 \%$ of cases annually [38].

Malaria is detected in all age groups, but the most affected group is the 20-29-year-old group (10,317 of 20,544 cases [50.22\%] in 2001-2017; Table 4). Malaria incidence in males $(17,140$ of 20,544 cases [83.43\%] in 2001-2017) was markedly higher than that in females $(3,404$ of 20,544, 16.57\%) (Table 4). Although there was a progressive decline in vivax malaria cases in individuals over 17 years of age from 2001-2017, the majority of cases were reported in young men 20-29 years of age (10,317 of 20,544 cases, 50.22\%) (Table 4). Males 40-49 years of age comprised the group with the second highest number of cases (3,051 of 20,544, 14.85\%) (Table 4). The notably higher case prevalence in 20-29-year-old males suggests an increase in the risk of infection outside their places of residence, as these males are the most mobile members of the population and are most likely to be working in the fields or serving as military personnel in Korea. This is consistent with the fact that human contact with vector mosquitoes and the distance between human residences and mosquito habitats are pivotal conditions determining the incidence of malaria [42].

\section{IMPORTED CASES}

The number of imported malaria cases has increased in Korea over the past 20 years (Table 1). Most cases of imported malaria were infections with $P$. falciparum or $P$. vivax. Infections with $P$. falciparum were mainly acquired in Africa, and vivax malaria infections mainly in Southeast Asia, consistent with the typical parasite frequencies of these areas. Imported malaria has a higher incidence in males than in females. Similar demographics have been reported in European countries [43-45]. Males in their 20s are most likely to be infected with imported malaria, likely because they are more likely to travel to areas with a high risk of malaria infection and participate in experiential tourism [43-45]. 


\section{MOSQUITO VECTOR}

A dramatic reduction in the incidence of vivax malaria has been achieved through large-scale national control systems in Korea. Yet, active transmission still persists, particularly in Incheon-si and northern part of the Gyeonggi-do. Control of the mosquito vector is essential for the eradication of malaria. Vector, pathogen, and host controls are the principal strategies of an effective malaria control program. The use of national media to increase the awareness of the need for personal protection against mosquitoes should also aid in transmission reduction.

Malaria is transmitted to humans by the bite of a female anopheline mosquito vector carrying malaria parasites during the acquisition of a blood meal. These vector populations depend on specific climatic and sociodemographic factors. $P$. vivax is transmitted in Korea by 6 Anopheles species (the Hyrcanus Group): An. sinensis sensu stricto, An. belerae, An. pullus, An. kleini, An. sineroides, and An. lesteri [46,47]. The Hyrcanus group of Anopheles mosquitoes typically dwells near irrigation ditches, ponds, ground pools, and uncultivated fields [48]. Studies of the infection capacity of An. sinensis, the most dominant Hyrcanus species in August, September, and October, and the primary vector of malaria in Korea, have been conducted [48-50]. However, an understanding of the other 5 species remains elusive [51]. The frequency of contact of An. sinensis with humans is very low because this vector is highly zoophilic [52,53]. Other factors reducing the contact are the widespread application of personal protection against mosquito bites [54] and the low to moderate longevity of An. sinensis in Korea [19,49]. Thus, the opportunity for malaria transmission by mosquitoes appears limited. In addition to the 6 major Anopheles species, An. koreicus and An. lindesayi japonicus (from the Barbirostris and Lindesayi group, respectively) have been reported in Korea [5558]. However, the incidence of malaria due to these 2 vectors has not been studied. It is presumed that these 2 species live mainly in swamps and stream margins, including stream inlets and pools [48,59], with low human-vector contact preventing human blood meals and P. vivax infection. However, there is no evidence that these species cannot develop or propagate malaria parasites. Additionally, no systematic reports have focused on the densities of the malaria vector mosquitoes as active disease vectors from 1993-2017. The only related report was issued by the KCDC, which chronicled the monitoring of malaria vector mosquito density and $P$. vivax malaria infections since 2009 [60]. From 2013-2014, the density of An. sinensis in- creased at most collection sites with the same high tendency of annual mean temperature and precipitation [61]. More research on malaria vectors in Korea is needed to provide detailed information about $P$. vivax transmission capacity. The hypnozoites that cause relapse may be activated weeks or months after the initial infection. Little is known about the biology and determinants of this latency [62].

\section{INCUBATION PERIOD OF $P$, vivax MALARIA}

In Korea, tertian malaria consists of 2 sub-groups. A non-latent group displays symptoms 10-21 days after infection (a short incubation period) [63]. A latent group displays symptoms 6-18 months after infection (a longer incubation period) with unusual biological characteristics and the presence of hypnozoites. In addition, the observed distribution of latent and non-latent infection reflects the adaptation of the parasite to the seasonal population dynamics of the An. sinensis vector, ensuring continued transmission of vivax malaria in this temperate zone despite a long winter period [63]. The occurrence of this long-term latency in vivax malaria in Korea over all four seasons impedes disease management at the national level.

In Korea, vivax malaria transmission is largely limited to the DMZ. Sixty to seventy percent of patients, including those diagnosed in the summer, are classified as long-term latency patients [38]. By monitoring travel routes of people living in non-endemic areas from 2000-2003, the mean duration of long-term latency was identified as 48.2 weeks (337.4 days), which was 12.7 times longer than that of short-term latency (26.6 days) [63]. The factors affecting long-term latency remain unclear, but it is presumed that temperature, infection time, and number of sporozoites play a role. It has also been hypothesized that differences in latency periods among populations may involve parasite genetics and/or the vector's capacity for sporozoites. It was recently reported by Goo et al. [64] that the genotype of PvMSP-1 is related to the duration of the latent period. The PuMSP-1 recombinant type was predominant in the long latent period samples, while the PuMSP-1 Sal1 type was most prevalent in the short latent period.

\section{FUTURE STRATEGIES FOR THE ELIMINATION OF VIVAX MALARIA IN KOREA}

Malaria has been an endemic disease in Korea for 25 years since its re-emergence in 1993, with a rapidly increasing trend 
up until 2000. Since then, there has been a mixed pattern of repeated increases and decreases in malaria outbreaks but a steadily declining trend in overall incidence. Academia, central government, local governments, and the military are carrying out various projects that include rapid patient discovery and treatment, and vector control with the basic goal of malaria elimination through treatment, intervention, and pathogen management. Korea is now reaching the pre-phase of malaria elimination, but outbreaks still continue. Additional efforts are needed for the complete elimination of malaria within the next few years and ongoing prevention.

Microscopic examination as a method to diagnose malaria has some limitations, but this method is regarded as the gold standard. Microscopic examination requires skilled specialists to prepare and examine blood smears. Furthermore, sample preparation and inspection are time-consuming. Polymerase chain reaction (PCR)-based diagnostic protocols have been recognized as powerful tools to detect malaria infection and differentiate the infected species with high specificity and sensitivity $[65,66]$. Nevertheless, PCR methods also have limitations, such as high cost and low applicability without adequate laboratory equipment. The rapid diagnostic test (RDT) has replaced microscopic examination in many malaria endemic countries worldwide because of its relatively reliable diagnostic performance and easy applicability. However, the RDT also has several drawbacks including its low sensitivity against low parasitemia blood samples. To overcome these shortcomings, a new tool that can detect very small amounts of antigen and antibody is needed. As shown in Table 1 and Fig. 3, similar numbers of vivax malaria cases were reported from 2014-2017, implying that a steady state of malaria transmission in Korea has been maintained in recent years. Therefore, efforts toward more intensive and systematic malaria surveillance, especially in Incheon-si and the northern part of Gyeonggi-do, are necessary to reduce the potential for future increases in malaria cases in endemic areas. The re-emergence of malaria at the DMZ in 1993 is regarded as an unstable malaria transmission that spontaneously appeared and disappeared within a short period of time without a clear reason [31]. To achieve the goal of complete malaria eradication in Korea, it is necessary to consider re-introduction of active case detection, as employed in the 1960s and 1970s.

In general, the circumsporozoite protein (CSP) antibody is produced by sensitization to the antigen presenting cell (APC) when the sporozoite circulates in the human body for 30 to
60 minutes. The CSP antibody has a short half-life that disappears before the next epidemic [67]. In the winter season, a survey for the presence of the CSP antibodies among the residents of high malaria risk areas in Paju-si, Gyeonggi-do, Cheolwon-gun, Gangwon-do, and Gangwha-gun, Incheon-si, might be useful for providing valuable and indirect data with other malarial antibodies for the certification of complete elimination of vivax malaria in Korea. In addition, new malaria management measures should be carried out in accordance with the situation in 2018.

\section{CONCLUSION}

The continued and systematic strengthening of control activities for vivax malaria in Korea is premised on the view that the number of vivax malaria cases has reached a steady-state level after its re-emergence in 1993. In spite of the good prospects for success, elimination has proceeded slowly and has been unsuccessful. The geographic boundary of transmission has not shrunk. These realities provide hints that technical aspects, such as resistance to the vector and drugs, are not primarily important and multiple factors are involved in an effective control system. With the current plateau in the number of malaria cases, more efforts must be made to ensure elimination of vivax malaria in Korea. Thus, the government and private sector have to collaborate in diverse areas including education, agriculture, environment, finance, research, medical inspection, communication, and transportation, and must implement all strategies that feasibly suppress the transmission of the infection. Although, in the past time, vivax malaria were considered to be mostly benign and rarely severe and Korea is one of successfully controlled nations for vivax malaria, the precise pathophysiology of $P$. vivax infection still remain poorly elusive and there is a critical aperture in the current knowledge on vivax malaria in Korea, for examples, drug resistance against chloroquine and primaquine that have been used for the treatment in Korea, genetic alterations in parasite's genomic contents, changes in host responses and incubation times etc. Coordinated multidisciplinary struggles are pivotal to bridge this knowledge aperture.

\section{ACKNOWLEDGMENTS}

This work was supported by the Research Grant from Inha University. 


\section{CONFLICT OF INTEREST}

The authors declare that they have no competing interests.

\section{REFERENCES}

1. Centers for Disease Control and Prevention. The history of malaria, an ancient disease. [Internet]; Available from: https://www. cdc.gov/malaria/about/history/. Accessed on 10 Jun 2017.

2. World Health Organization. World Malaria Report, 2017. Geneva, Switzerland. World Health Organization. 2017.

3. Mendis K, Sina BJ, Marchesini P, Carter R. The neglected burden of Plasmodium vivax malaria. Am J Trop Med Hyg 2001; 64: $97-$ 106.

4. Carlton JM, Sina BJ, Adams JH. Why is Plasmodium vivax a neglected tropical disease? PLoS Negl Trop Dis 2011; 5: e1160.

5. Yekutiel P. Eradication of Infectious Diseases: A Critical Study. Basel, Switzerland. S Karger AG. 1980, pp 34-88.

6. White NJ. Determinants of relapse periodicity in Plasmodium vivax malaria. Malaria J 2011; 10: 297.

7. Betuela I, Rosanas-Urgell A, Kiniboro B, Stanisic D, Samol L, et al. Relapses contribute significantly to risk of $P$. vivax infection and disease in Papua New Guinean children 1-5 years of age. J Infect Dis 2012; 206: 1771-1780.

8. Chu JK. A century history of parasitology in Korea. Seoul Korea. Kookje Kyoyok Munhwa 1986; 1-144 (Monograph in Korean).

9. Chun CH, Kim JJ. Malaria in Korea. Korean Med 1959; 2: 63-66.

10. Kim DC. Status of malaria infection in the Republic of Korea. Yonsei Rep Trop Med 1982; 13: 59-62.

11. Kwak YG, Lee HK, Kim M, Um TH, Cho CR. Clinical characteristics of vivax malaria and analysis of recurred patients. Infect Chemother 2013; 45: 69-75.

12. Hasegawa Y. Malaria in Korea. J Chosen Med Soc 1913; 4: 53-69.

13. Chai JY. Re-emerging Plasmodium vivax malaria in the Republic of Korea. Korean J Parasitol 1999; 37: 129-143.

14. Gennis J, Straus B, Kenney M, Klein B. The use of primaquine for the treatment of malaria in Korean veterans. Am J Med 1954; 17: 223-228.

15. National Malaria Eradication Service, Ministry of Health and Social Affairs, Republic of Korea. Malaria pre-eradication programme in Korea, 1961-1965. Progress Report. 1966. 44-70 (in Korean).

16. Paik $\mathrm{YH}$, van der Gugten AC. Evaluation report on the results of the passive case detection conducted in the Korea malaria preeradication programme during the period 1960-1965. Korean J Parasitol 1996; 4: 1-9.

17. Chow CY. Arthropods of public health importance in Korea. Korean J Entomol 1973; 3: 31-54.

18. Soh CT, Lee KT, Im KI, Min DY, Ahn MH, Kim JJ, Yong TS. Current status of malaria in Korea. Yonsei Rep Trop Med 1985; 16 : 11-18.

19. Paik YH, Ree HI, Shim JC. Malaria in Korea. Jpn J Exp Med 1988; 58: 55-56.
20. World Health Organization. Synopsis of the world malaria situation in 1979. Wkly Epidemiol Rec 1981; 56: 145-149.

21. Chai IH, Lim GI, Yoon SN, Oh WI, Kim SJ, Chai JY. Occurrence of tertian malaria in a male patient who has never been abroad. Korean J Parasitol 1994; 32: 195-200 (in Korean).

22. Feachem RGA, Phillips AA, Hwang J, Cotter C, Wielgosz B, Greenwood BM, Sabot O, Rodriguez MH, Abeyasinghe RR, Ghebreyesus TA, Snow RW. Shrinking the malaria map: Progress and prospects. Lancet 2010; 376: 1566-1578.

23. Lee KJ, Kim CB, Choi BJ, Park KH, Park JK. Analysis of vivax malaria cases in Gangwon-do (province), Korea in the year 2000. Korean J Parasitol 2001; 39: 301-306.

24. Moon JJ, Cho SY. Incidence patterns of vivax malaria I civilians residing in a high-risk county of Kyonggi-do (province), Republic of Korea. Korean J Parasitol 2001; 39: 293-299.

25. Lee JS, Lee WJ, Cho SH, Ree HI. Outbreak of vivax malaria in areas adjacent to the demilitarized zone, South Korea. Am J Trop Med Hyg 2002; 66: 13-17.

26. Klein TA, Pacha LA, Lee HC, Kim HC, Lee WJ, Lee JK, Jeung GG, Sames WJ, Gaydos JC. Plasmodium vivax malaria among U.S. forces Korea in the Republic of Korea, 1993-2007. Mil Med 2009; 174: 412-418.

27. Yeom JS, Kim TS, Oh S, Sim JB, Barn JS, Kim HJ, Kim YA, Ahn SY, Shin MY, Yoo JA, Park JW. Plasmodium vivax malaria in the Republic of Korea during 2004-2005: changing patterns of infection. Am J Trop Med Hyg 2007; 76: 865-868.

28. Park JW, Jun G, Yeom JS. Plasmodium vivax malaria: status in the Republic of Korea following reemergence. Korean J Parasitol 2009; 47 (suppl): 39-50.

29. Korean Centers for Diseases Control and Prevention. Guidelines for Malaria Prevention and Control 2016. 2016, pp 1-177 (in Korean).

30. Yeom JS, Ryu SH, Oh S, Choi DH, Song KJ, Oh YH, Lee JH, Kim YA, Ahn SY, Yang HY, Cha JE, Park JW. Evaluation of anti-malarial effects of mass chemoprophylaxis in the Republic of Korea Army. J Korean Med Sci 2005; 20: 707-712.

31. Ree HI. Unstable vivax malaria in Korea. Korean J Parasitol 2000; 38: $119-138$.

32. Thimasarn K. Updates on malaria elimination in DRP Korea. Brisbane: Asia Pacific Malaria Elimination Network Inaugural Meeting 2009.

33. Korean Centers for Disease Control and Prevention. Effectiveness and safety of primaquine chemoprophylaxis against $P$. vivax before transmission season. Report of Bi-Regional Meeting for Control of Malaria in East Asia 2005; 8: 17-20.

34. Pinyowiwat V. Democratic People's Republic of Korea Malaria Report. World Health Organization Regional Office for Southeast Asia Malaria Report 2004.

35. Cho SH, Lee HW, Shin EH, Lee HI, Lee WG, Kim CH, Kim JT, Lee JS, Lee WJ, Jung GG, Kim TS. A mark-release-recapture experiment with Anopheles sinensis in the northern part of Gyeonggi-do, Korea. Korean J Parasitol 2002; 40: 139-148.

36. Iwagami M, Hwang S-Y, Fukumoto M, Hayakawa T, Tanabe K, 
Kim SH, Kho WG, Kano S. Geographical origin of Plasmodium vivax in the Republic of Korea: haplotype network analysis based on the parasite's mitochondrial genome. Malaria J 2010; 9: 184.

37. Korean Centers for Diseases Control and Prevention. Infectious Diseases Surveillance System. [Internet]; Available from: http:// is.cdc.go.kr/.

38. Kim HC, Pacha LA, Lee WJ, Lee JK, Gaydos JC, Sames WJ, Lee HC, Bradley K, Jeung GG, Tobler SK, Klein TA. Malaria in the Republic of Korea, 1993-2007. Variables related to re-emergence and persistence of Plasmodium vivax among Korea populations and U.S. forces in Korea. Mil Med 2009; 174: 762-769.

39. Srimath-Tirumula-Peddinti RC, Neelapu NR, Sidagam N. Association of climatic variability, vector population and malarial disease in district of Visakhapatnam, India: A modeling and prediction analysis. PLoS One 2015; 10: e0128377.

40. Lee SH, Nam KW, Jeong JY, Yoo SJ, Koh YS, Lee S, Heo ST, Seong SY, Lee KH. The effects of climate change and globalization on mosquito vectors: evidence from Jeju Island, South Korea on the potential for Asian tiger mosquito (Aedes albopictus) influxes and survival from Vietnam rather than Japan. PLoS One 2013; 8: e68512.

41. Oliver SV, Brooke BD. The effect of elevated temperatures on the life history and insecticide resistance phenotype of the major malaria vector Anopheles arabiensis (Diptera: Culicidae). Malaria J 2017; 16: 73.

42. Kim JH, Park SY. The impact of land use structure and vector habitat conditions on the incidence of malaria - A case study in high-incidence areas. J Korean Assoc Geographic Inform Studies 2013; 16: 12-24 (in Korean).

43. Baas MC, Wetsteyn JC, van Gool T. Patterns of imported malaria at the academic medical center. Amsterdam, the Netherlands. J Travel Med 2006; 13: 2-7.

44. Siikamäki H, Kivelä P, Lyytikäinen $\mathrm{O}$, Kantele A. Imported malaria in Finland 2003-2011: prospective nationwide data with rechecked background information. Malaria J 2013; 12: 93.

45. Rey S, Zuza I, Martinez-Mondejar B, Rubio JM, Merino FJ. Imported malaria in an area in southern Madrid, 2005-2008. Malaria J 2010; 9: 290.

46. Hwang UW. Revisited ITS2 phylogeny of Anopheles (Anopheles) Hyrcanus group mosquitoes: reexamination of unidentified and misidentified ITS sequences. Parasitol Res 2007; 101: 885-894.

47. Shin EH. Characteristics of malaria vector mosquitoes in the Republic of Korea. Public Health Weekly Report KCDC 2014; 7: 813-818 (in Korean).

48. Rueda, LM, Brown TL, Kim HC, Chong ST, Klein TA, Foley DH, Anyamba A, Smith M, Pak EP, Wilkerson RC. Species composition, larval habitats, seasonal occurrence and distribution of potential malaria vectors and associated species of Anopheles (Diptera: Culicidae) from the Republic of Korea. Malaria J 2010; 9: 55.

49. Rueda LM, Kim HC, Klein TA, Pecor JE, Li C, Sithiprasasna R, Debboun M, Wilkerson. Distribution of larval habitat characteristics of Anopheles Hyrcanus Group and related mosquito species (Diptera: Culicidae) in South Korea South Korea. J Vector Ecol
2006; 31: 198.

50. Yoo DH, Shin EH, Park MY, Kim HC, Lee DK, Lee HH, Kim HK, Chang KS. Mosquito species composition and Plasmodium vivax infection rates for Korean Army bases near the Demilitarized Zone in the Republic of Korea, 2011. Am J Trop Med Hyg 2013; 88: 2428.

51. Lee HW, Cho SH, Shin EH, Lee JS, Lee JS, Chai JY, Lee SH, Kim TS. Experimental infection of Anopheles sinensis with Korean isolates of Plasmodium vivax. Korean J Parasitol 2001; 39: 177-183.

52. Ree HI, Hong HK, Paik YH. Daily survival and human blood index of Anopheles sinensis, the vector species of malaria in Korea. J Am Mosquito Control Assoc 2001; 17: 67-72.

53. Ree HI. Studies on Anopheles sinensis, the vector species of vivax malaria in Korea. Korean J Parasitol 2005; 43: 75-92.

54. Paik YH, Song JH, Ree HI, Hong HK. Epidemiological studies on malaria situation in Korea. Part 1. On the bionomics of Anopheles sinensis and its relation to malaria in Korea. New Med 1965; 8: 1043-1049.

55. Foley DH, Klein TA, Kim HC, Sames WJ, Wilkerson RC, Rueda LM. Geographic distribution and ecology of potential malaria vectors in the Republic of Korea. J Med Entomol 2009; 46: 680692.

56. Tanaka K, Mizusawa K, Sauhstad ES. A revision of the adult and larval mosquitoes of Japan (including the Ryukyu Archipelago and the Ogasawara Islands and Korea (Diptera: Culicidae). Contrib Amer Ent Inst 1979; 16: 1-987.

57. Hwang UW. Revisited ITS2 phylogeny of Anopheles (Anopheles) Hyrcanus Group mosquitoes: Reexamination of Unidentified and misidentified ITS2 sequences. Parasitol Res 2007; 101: 885894.

58. Rueda LM, Li C, Kim HC, Klein TA, Foley DH, Wilkerson RC. Anopheles belenrae, a potential vector of Plasmodium vivax in the Republic of Korea. J Am Mosq Control Assoc 2010; 26: 430-432.

59. Kim HC, Sames WJ, Chong ST, Lee IY, Lee DK, Kim HD, Rueda LM, Klein TA. Overwintering of Anopheles lindesayi larvae in the Republic of Korea. J Am Mosquito Control Assoc 2009; 25: 3237.

60. Lee HS, Roh JY, Joo YR. Monitoring of malaria vector mosquitoes and their Plasmodium vivax infection in the Republic of Korea, 2015. Public Health Weekly Report KCDC 2015; 9: 382-387 (in Korean).

61. Lee HW, Park SH, Na BK, Kim HC, Klein TA, Jeon BY, Shin EH, Bahk YY, Suh HH, Kim TS, Chung MK. Changing vivax malaria transmission features in the Republic of Korea during 20132014. J Trop Dis 2017; 5: e1000231.

62. Chu CS, White NJ. Management of relapsing Plasmodium vivax malaria. Expert Rev Anti Infect Ther 2016; 14: 885-900.

63. Nishiura H, Lee HW, Cho SH, Lee WG, In TS, Moon SU, Chung GT, Kim TS. Estimate of short and long-term incubation periods of Plasmodium vivax malaria in the Republic of Korea. Trans $\mathrm{R}$ Soc Trop Med Hyg 2007; 101: 338-343.

64. Goo YK, Moon JH, Ji SY, Chung DI, Hong Y, Cho SH, Lee WJ, Kim JY. The unique distribution of the Plasmodium vivax mero- 
zoite surface protein 1 in parasite isolates with short and long latent periods from the Republic of Korea. Malaria J 2015; 14: 299.

65. Mekonnen SK, Aseffa A, Medhin G, Berhe N, Velavan TP. Reevaluation of microscopy confirmed Plasmodium falciparum and Plasmodium vivax malaria by nested PCR detection in southern Ethiopia. Malaria J 2014; 13: 48.

66. Kang JM, Cho PY, Moe M, Lee J, Jun H, Lee HW, Ahn SK, Kim TI, Pak JH, Myint MK, Lin K, Kim TS, Na BK. Comparison of the diagnostic performance of microscopic examination with nested polymerase chain reaction for optimum malaria diagnosis in Upper Myanmar. Malaria J 2017; 16: 119.

67. Cho PY, Lee SW, Ahn SK, Kim JS, Cha SH, Na BK, Park YK, Lee SK, Lee WJ, Nam HW, Hong SJ, Pak JH, Kang YJ, Sohn YS, Bahk YY, Cho HI, Kim TS, Lee HW. Evaluation of circumsporozoite protein of Plasmodium vivax to estimate its prevalence in the Republic of Korea: an observational study of incidence. Malaria J 2013; $12: 448$. 
